\title{
Administration of an Aldose Reductase Inhibitor Induces a Decrease of Collagen Fluorescence In Diabetic Rats
}

Gerardo Suárez, Rama Rajaram, Kailash C. Bhuyan, “ Arnold L. Oronsky, and Jo Alene Goidı

Milton and Miriam Petrie Arthritis Research Laboratory, Department of Orthopaedics, and *Department of Ophthalmology, Mount Sinai School of Medicine, New York, New York 10029; ${ }^{\ddagger}$ Medical Research Division, Lederle Laboratories,

Division of American Cyanamid Company, Pearl River, New York 10965

\begin{abstract}
As a consequence of an increased flux through the sorbitol pathway fructose levels rise in various tissues in diabetes. Also, in vitro nonenzymatic fructosylation of protein induces the generation of fluorescence at a rate 10 times greater than glucosylation. The administration of sorbinil, an aldose reductase inhibitor known to lower tissue fructose concentration, to experimental diabetic rats led to a decrease in the fluorescence related to advanced Maillard products in their skin collagen. This effect is consistent with the in vivo occurrence of nonenzymatic fructosylation of collagen. A potential pathogenetic role for this posttranslational modification in diabetic complications should be considered.
\end{abstract}

\section{Introduction}

Despite significant improvements in the control of blood glucose levels by diet or drug therapy the long-term complications of diabetes are still leading causes of death and morbidity (1, 2). Among the various biochemical processes that have been proposed as underlying the pathogenesis of these complications, nonenzymatic glucosylation ${ }^{1}$ of proteins has been the focus of increasing attention during the last decade (3-5). The term nonenzymatic glycosylation collectively denotes a multireaction pathway, also termed the Maillard reaction, whereby reducing sugars react with protein amino groups and form Schiff base-mediated adducts that rearrange into more stable glycoconjugates. If the sugar is glucose the rearrangement is known as the Amadori rearrangement and the glycoconjugate is a ketoamine-linked 1-deoxy hexose. The products of the rearrangement undergo further transformations which lead to the emergence of poorly characterized fluorescent structures. Recent evidence (6) supports the view that these

A preliminary account of these results has been presented in Kashikojima, Japan (Suárez, G., R. Rajaram, K. Bhuyan, A. L. Oronsky, and J. A. Goidl. 1986. International Symposium on Polyol Pathway and its Role in Diabetic Complications. 38).

Address all correspondence to Dr. Gerardo Suarez, Department of Biochemistry, New York Medical College, Valhalla, NY 10595.

Received for publication 8 June 1987 and in revised form 22 February 1988.

1. Throughout the text the word "glycosylation" has been employed to denote the nonenzymatic protein modification by any sugar whereas "glucosylation" denotes exclusively the reaction of glucose with protein.

J. Clin. Invest.

(c) The American Society for Clinical Investigation, Inc. 0021-9738/88/08/0624/04 \$2.00

Volume 82, August 1988, 624-627 fluorescent moieties mediate the establishment of crosslinks between protein molecules which may underlie some pathogenetic events in the complications of diabetes (7).

Alternative hypotheses to explain the complications of diabetes are based on the operation of the sorbitol pathway. This is a metabolic shunt, the net result of which is the conversion of glucose to fructose (Scheme I) with the formation of sorbitol as an intermediate metabolite. Reduction of glucose is catalyzed by the NADPH-requiring enzyme aldose reductase (alditol: NADP oxidoreductase, E.C. 1.1.1.21). The resulting sorbitol can be oxidized enzymatically to fructose through the action of sorbitol dehydrogenase (L-iditol:NAD oxidoreductase, E.C. 1.1.1.14), with NAD+ serving as a coenzyme. An explanation of the long-term complications of diabetes such as cataracts and peripheral neuropathy $(8,9)$ is based on the accumulation of sorbitol within cells. Sorbitol accumulation occurs because the cell membrane is impermeable to sorbitol, and thus, a higher flux through the sorbitol pathway, as in diabetes, would result in a rise in the intracellular level of the polyol. This, in turn, would induce an increase in the osmotic pressure leading to the transfer of water to the intracellular compartment, ionic leakage, and cell damage.

In contrast to the role of sorbitol the second step in the sorbitol pathway, the generation of fructose, has received, to our knowledge, almost no attention although fructose levels have been shown to increase up to 23-fold in tissues of diabetic animals where the sorbitol pathway is active (10). Numerous other studies have documented elevated intracellular concentrations of fructose in diabetic animals (11-13). The concept that the sorbitol pathway might mediate diabetic complications by mechanisms other than those related to the "osmotic hypothesis" described above was suggested to us as a result of considerations of the following experimental findings: incubation of BSA with $0.5 \mathrm{M}$ fructose led to the generation of protein-bound fluorescent products at a rate 10 times greater than when glucose was used as the glycosylating sugar under the same conditions (14). We, therefore, concluded that fructose was a much more efficient glycosylating sugar than glucose. Based on this we hypothesized that nonenzymatic fructosylation might occur in vivo and, if so, might mediate complications of diabetes that depend on the operation of the sorbitol pathway.

To test this hypothesis a study was undertaken that compared protein-bound fluorescence in skin from diabetic rats treated with the aldose reductase inhibitor, sorbinil $([+] 6-$ fluoro-spiro-[chroman-4, $4^{1}$-imidazolidine]-2 $2^{1}, 5^{1}$-dione), with that found in the skin of untreated diabetic animals. It was conjectured that protein-bound fluorescence resulting from nonenzymatic fructosylation would be affected by sorbinil but not that which results from glucosylation, since this treatment would have no effect on the glucose levels $(12,15,16)$. 


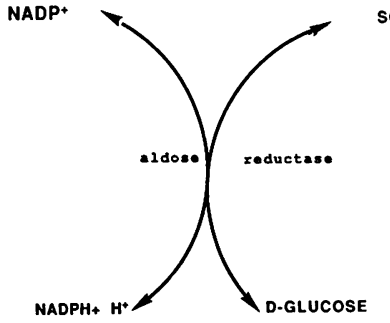

SORBITOL

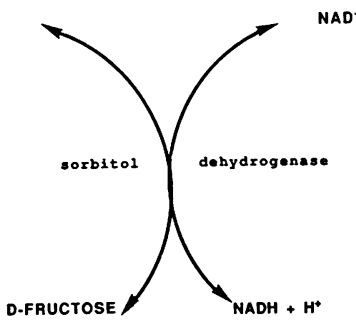

Scheme 1 .

\section{Methods}

Two groups of male Sprague-Dawley rats weighing an average of $160 \mathrm{~g}$ were rendered diabetic by a single subcutaneous injection of streptozotocin $(80 \mathrm{mg} / \mathrm{kg}$ body weight). One of these groups of diabetic rats was fed a diet supplemented with sorbinil $(400 \mathrm{mg} / \mathrm{kg}$ of rodent laboratory chow), which was kindly provided by Pfizer, Inc., Groton, CT. After $56 \mathrm{~d}$ of treatment the rats were killed by cervical dislocation immediately after withdrawal of blood from the retrobulbar blood vessels.

Solubilization of skin collagen by bacterial collagenase. The skin of each animal was removed by careful dissection and stored frozen at $-80^{\circ} \mathrm{C}$ until used. Portions of skin were cut into small pieces and washed twice with $0.05 \mathrm{M}$ phosphate, $\mathrm{pH} 7.4$, containing $0.1 \mathrm{M} \mathrm{NaCl}$ (PBS), $0.01 \mathrm{M} N$-ethylmaleimide and $0.01 \mathrm{M}$ EDTA by slow overnight stirring and subsequent decantation. After removal of hair the residue was suspended in $10 \mathrm{ml}$ PBS and homogenized with a Polytron homogenizer at $4^{\circ} \mathrm{C}$. The homogenate was centrifuged at $10,000 \mathrm{~g}$ for 10 min at $4^{\circ} \mathrm{C}$ and the supernatant discarded. The pellet was washed with $30 \mathrm{ml}$ of cold water three times. To each washed pellet $200 \mathrm{ml}$ of a 1:1, vol:vol, ethanol/ether mixture was added and stirred overnight. The ethanol/ether extract was discarded and the residue was washed with water by centrifugation at $12,000 \mathrm{~g}$. The washed pellet was resuspended in $5 \mathrm{ml}$ of $0.05 \mathrm{M}$ borate, $\mathrm{pH} 7.4$, containing $1 \mathrm{M} \mathrm{NaCl}$ for preequilibration, and resuspended in $2 \mathrm{ml}$ of a mixture containing 0.05 $\mathrm{M}$ borate, $\mathrm{pH} 7.4,50 \mathrm{mM} \mathrm{CaCl}_{2}, 20 \mathrm{mM}$-ethylmaleimide and $155 \mathrm{U}$ of Clostridium histolyticum collagenase, form III (Advance Biofactures, Lynbrook, NY). Digestion was achieved by stirring slowly overnight at $37^{\circ} \mathrm{C}$. The incubation mixture was centrifuged at $12,000 \mathrm{~g}$ for $30 \mathrm{~min}$ and assays were conducted in supernatant aliquots. Fluorescence measurements were carried out in a recording spectrofluorometer (204 A; Perkin-Elmer Corp., Pomona, CA) using an excitation wavelength of $350 \mathrm{~nm}$, an emission wavelength of $410 \mathrm{~nm}$, and slit width of $10 \mathrm{~nm}$ for both excitation and emission. The instrument was adjusted so that the emission of the standard No. 4 from the manufacturers (tetraphenyl butadiene) was 70 at a photomultiplier gain of 3 and a sensitivity of 0.3 . Fluorescence values were normalized with respect to the hydroxyproline content of the collagenase digest as determined according to Woessner (17). Fluorescence measurements of all the samples were conducted on a single day.
Incubation of BSA with sugars. BSA (fatty acid-free, Sigma Chemical Co., St. Louis, MO), used as a model protein, was incubated at a concentration of $6 \mathrm{mg} / \mathrm{ml}$ in $0.05 \mathrm{M}$ sodium phosphate $0.1 \mathrm{M} \mathrm{NaCl}$, $\mathrm{pH} 7$ (PBS), containing either $0.5 \mathrm{M}$ glucose or $0.5 \mathrm{M}$ fructose at $37^{\circ} \mathrm{C}$ for $32 \mathrm{~d}$ under sterile conditions in a humidified atmosphere. Subsequently, the incubation mixtures were dialyzed vs. $400 \mathrm{vol}$ of PBS for 4 $d$ with several changes of the effusate. Then, the dialyzed protein solutions were centrifuged at $10,000 \mathrm{~g}$ at $4^{\circ} \mathrm{C}$ for $20 \mathrm{~min}$, the supernates were passed through ultrafiltration membranes (Millipore/Continental Water Systems, Bedford, MA) and stored at $4^{\circ} \mathrm{C}$. The sterile solutions were precipitated with $5 \mathrm{vol}$ of cold tetrahydrofuran and the precipitates were collected by centrifugation at $3,500 \mathrm{~g}$ for $10 \mathrm{~min}$ and washed three times with tetrahydrofuran. The washed precipitates were suspended in PBS and dialyzed vs. 100 vol of PBS with four changes of the effusate.

Fluorescence emission spectra. Collagenase digests obtained as described in the legend to Fig. 1 were scanned in the Perkin-Elmer spectrofluorometer. Full pen deflection corresponded to $1 \mathrm{~V}$. Excitation wavelength was $350 \mathrm{~nm}$. All samples were taken to a concentration of hydroxyproline of $400 \mu \mathrm{g} / \mathrm{ml}$.

Nonenzymatically glycosylated BSA solutions, obtained after incubation with sugars as described above, were taken to a protein concentration of $0.365 \mathrm{mg} / \mathrm{ml}$ (as determined by the Lowry method) and the emission spectra taken as described.

\section{Results}

Relevant blood glucose values and body weight changes of the rats are summarized in Table I. Control rats gained a mean of $150.8 \mathrm{~g}$ during the period of observation while diabetic rats lost a mean of $16.18 \mathrm{~g}$ (untreated) and $22.19 \mathrm{~g}$ (sorbinil-treated).

Fig. 1 shows the results on the fluorescence associated with the material that was solubilized from the skin by purified bacterial collagenase. Preliminary surveys on samples from 50 rats revealed that the solubilized material had an amino acid composition almost identical to that of type I collagen obtained from rat skin according to the method of Chandrakasan et al. (18). A minor proportion of cysteine indicated that a small percentage of type III collagen (19), also present in the skin, was likewise solubilized. In the same preliminary study no significant differences were found in the yield of collagenase-released hydroxyproline-containing material between diabetic and control animals. Control rat skin yielded 94.2 222.44 SD nmol of hydroxyproline/mg wet tissue, while diabetic rat skin yielded $90.97 \pm 21.46$ SD nmol (control vs. diabetic $P$ $<0.6$ ). The normalized fluorescence associated with skin collagen of the untreated diabetic rats $(117 \pm 7)$ was almost twice that of control rats $(63 \pm 5)$. After treatment with sorbinil fluorescence was significantly $(P<0.003)$ reduced $(87 \pm 4)$.

Table I. Data on Experimental Animals

\begin{tabular}{|c|c|c|c|c|}
\hline \multirow[t]{2}{*}{ Experimental group } & \multicolumn{2}{|c|}{ Plasma glucose (mean $\pm \mathrm{SE})$} & \multicolumn{2}{|c|}{ Body weight (mean $\pm \mathrm{SE}$ ) } \\
\hline & & & & \\
\hline Days after streptozotocin injection & 3 & 56 & 0 & 56 \\
\hline \multirow[t]{2}{*}{ Control } & $135.6 \pm 2.3 \mathrm{mg} / \mathrm{dl}$ & ND & $162.2 \pm 1.3$ & $313 \pm 11.03$ \\
\hline & $n=12$ & & $n=12$ & $n=12$ \\
\hline \multirow[t]{2}{*}{ Diabetic, untreated } & $513.9 \pm 6.4 \mathrm{mg} / \mathrm{dl}$ & $515.8 \pm 15.4 \mathrm{mg} / \mathrm{dl}$ & $159.6 \pm 1.1$ & $143.4 \pm 6.2$ \\
\hline & $n=14$ & $n=14$ & $n=14$ & $n=14$ \\
\hline \multirow[t]{2}{*}{ Diabetic, sorbinil-treated } & $534.1 \pm 9.4 \mathrm{mg} / \mathrm{dl}^{*}$ & $494.1 \pm 28.2 \mathrm{mg} / \mathrm{dl}^{\ddagger}$ & $157.6 \pm 1.3$ & $129.5 \pm 3.6^{\S}$ \\
\hline & $n=14$ & $n=14$ & $n=14$ & $n=12$ \\
\hline
\end{tabular}

$n$ may differ from number of animals at the start of the experiment due to death or inadequate sample. * Not significantly different from untreated diabetic rats at $P \leq 0.084$. ${ }^{\ddagger}$ Not significantly different from untreated diabetic rats $P \leq 0.5$. $\$$ Not significantly different from untreated diabetic rats at $P \leq 0.077$. 


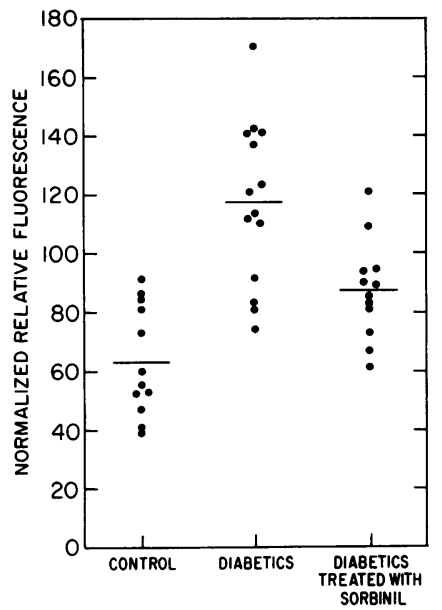

Figure 1. Normalized relative fluorescence of collagenase-solubilized material of normal and diabetic rats. Fluorescence emission (excitation wave length, $350 \mathrm{~nm}$ ) was measured in samples of solubilized skin obtained as described under Methods. Mean values were $63.7,87.6$, and 117.2 for the control, sorbinil-treated diabetic and diabetic group of rats, respectively. $P<0.003$ for the diabetic vs. sorbiniltreated diabetic group.

Fluorescence spectra were analyzed to characterize the fluorophors associated with collagen. For this purpose samples were chosen with a fluorescence emission closest to the mean value of the respective experimental group. Fig. 2 illustrates the emission spectra of the collagen solubilized from the skin of normal, diabetic, and sorbinil-treated diabetic rats. Sorbinil treatment led to a diminished emission maximum, compared with untreated diabetic rats, while the qualitative features of the spectra remained unaltered. Furthermore, the emission spectra of the collagenase-solubilized material was almost indistinguishable from the one obtained in the BSA samples that had been incubated with fructose at $37^{\circ} \mathrm{C}$ for $32 \mathrm{~d}$.

Fig. 2 also illustrates the fact that although the fluorescence of nonenzymatically fructosylated BSA was qualitatively undistinguishable from that of the nonenzymatically glucosylated protein, as is the case for collagen, its relative intensity was much higher (about 10-fold).

\section{Discussion}

From these data it was obvious that sorbinil treatment induced a highly significant reduction in collagen-bound fluorescence

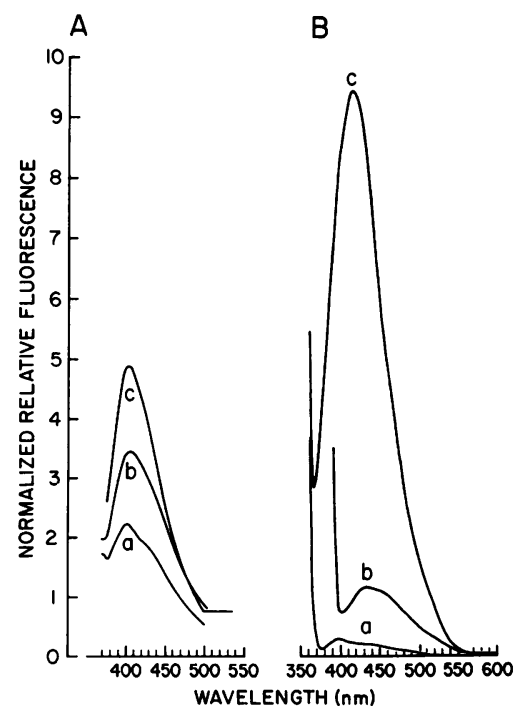

Figure 2. (A) Fluorescence emission spectra of rat skin samples solubilized by collagenase. Samples were obtained as described in the legend to Fig. 1. $a$, control; $b$, sorbinil-treated diabetic; $c$, diabetic. With the instrument settings corresponding to the measurements referred to in Fig. 1, samples a, $b$, and c showed normalized emission values of 60,88 , and 113 , respectively. (B) Fluorescence emission spectra of nonenzymatically glycosylated BSA. BSA was incubated for $32 \mathrm{~d}$

at $37^{\circ} \mathrm{C}$ without (a) sugar, $(b)$ or in the presence of $0.5 \mathrm{M}$ glucose, $(c)$ or fructose. Protein concentration in each sample was $0.365 \mathrm{mg} / \mathrm{ml}$. (See text for details of the preparations.) related to the later stages of the Maillard reaction. The dose of sorbinil used in this study has previously been shown to decrease fructose levels in tissues where the sorbitol pathway is active $(12,15,16)$. Since the inhibitor did not affect the blood glucose levels of the diabetic rats (Table I), as previously reported $(12,15,16)$, the decreased collagen fluorescence cannot be explained by a reduction in the extent of nonenzymatic glucosylation. The results are, however, consistent with a decreased generation of fluorophors that arise from nonenzymatic fructosylation of proteins. It should be noted, however, that administration of aldose reductase inhibitors has been shown to decrease sorbitol values more efficiently than fructose levels $(12,15,16)$. This may explain, in part, the inability of sorbinil to reverse collagen-associated fluorescence completely. Another explanation for the incomplete reversal of the high collagen fluorescence after sorbinil treatment is the probable coexistence of nonenzymatic glucosylation and fructosylation, which would be expected to occur under conditions of hyperglycemia uncorrected by sorbinil treatment. As already mentioned, nonenzymatic glucosylation also leads to the generation of protein-bound fluorescent moieties.

The extent of nonenzymatic glycosylation, which is a very slow reaction, for a given protein is largely dependent on its half-life. Thus, it could be hypothesized that the decreased fluorescence induced by sorbinil is a reflection of increased collagen turnover. This is highly improbable, based on the almost identical growth curves of the two groups of diabetic rats and on the documented lack of effect of sorbinil on collagen synthesis (20).

These considerations have led us to the view that our results provide presumptive evidence for the occurrence of nonenzymatic fructosylation in vivo. It has been proposed that aldose reductase inhibitors might have beneficial effects through a mechanism that involves a replenishment of reduced glutathione, which would lead to decreased disulfide bridge-mediated protein crosslinks upon nonenzymatic glycosylation (21). Fluorophors resulting from nonenzymatic glucosylation, however, appear to be generated at a higher rate under nonoxidative conditions (22), so our results cannot be satisfactorily explained by this hypothesis if fructosylation proceeds by analogous mechanisms.

The sorbitol pathway is generally thought to be an intracellular pathway, while the present study deals with the structural modification of collagen, which is an extracellular protein in its mature form. However, a recent report proposed extracellular sorbitol accumulation as a pathogenetic determinant in the syndrome of limited joint mobility (23), one of the sequelae of long-standing juvenile diabetes (24). Increased extracellular polyol concentration would lead to a higher degree of collagen hydration, which might underlie the pathophysiology of virtually all the complications of diabetes in collagencontaining organs. The general validity of the "collagen hydration" hypothesis $(25,26)$ must be questioned since nerve water content of peripheral nerve has been found unaltered in experimental diabetic rats (27). Also, the nonenzymatic fructosylation concept has the potential to provide an explanation of the pathology associated not only with collagenous proteins but also with noncollagenous proteins, such as lens crystallins and red cell membrane proteins, as well. Although fructose is generated intracellularly, there is evidence that significant diffusion of the sugar into the culture medium occurs in organ culture of aortas (28). Also, a recent report (29) indicated that 
sorbinil was effective at decreasing the concentration of sorbitol in human endothelial cells cultured in the presence of high glucose concentrations. This finding demonstrates that the sorbitol pathway is present in all kinds of vascular tissue and that the levels of sorbitol, and presumably of fructose, can be modulated by sorbinil. Leakage of fructose from vascular tissue, where the sorbitol pathway is active, to the extracellular collagenous matrix of the skin would be favored by the high degree of vascularization of this tissue (30).

In summary, our results are compatible with the in vivo occurrence of protein nonenzymatic fructosylation and warrant further exploration aimed at providing direct evidence of this posttranslational modification, such as identification of the corresponding advanced Maillard reaction products. If nonenzymatic fructosylation represents a major event, then the value of protein-bound fluorescence as a clinical marker (31) and its pathophysiological significance should be reassessed.

\section{Acknowledgments}

Supported in part by American Heart Association grant 82-943, and by the Milton and Miriam Petrie Fund and Lederle Laboratories.

\section{References}

1. Krolewski, A. S., J. H. Warram, and A. R. Christlieb. 1985. Onset, course, complications, and prognosis of diabetes mellitus. In Joslin's Diabetes Mellitus. A. Marble, L. P. Krall, R. F. Bradley, A. R. Christlieb, and J. S. Soeldner, editors. Lea and Febiger, Philadelphia. 251-277.

2. Entmacher, P., L. P. Krall, and S. N. Kranczer. 1985. Diabetes mortality from vital statistics. In Joslin's Diabetes Mellitus. A. Marble, L. P. Krall, R. F. Bradley, A. R. Christlieb, and J. S. Soeldner, editors. Lea and Febinger, Philadelphia.

3. Cerami, A., V. J. Stevens, and V. M. Monnier. 1979. Role of nonenzymatic glycosylation in the development of the sequelae of diabetes mellitus. Metab. Clin. Exp. 28(Suppl. 1):431-437.

4. Kennedy, L., and J. W. Baynes. 1984. Non-enzymatic glycosylation and the chronic complications of diabetes: an overview. Diabetologia. 26:93-98.

5. Brownlee, M., H. Vlassara, and A. Cerami. 1984. Nonenzymatic glycosylation and the pathogenesis of diabetic complications. Ann. Intern. Med. 101:527-537.

6. Brownlee, M., H. Vlassara, A. Kooney, P. Ulrich, and A. Cerami. 1986. Aminoguanidine prevents diabetes-induced arterial wall protein cross-linking. Science (Wash. DC). 232:1629-1632.

7. Schnider, S. L., and R. R. Kohn. Effects of age and diabetes mellitus on the solubility and nonenzymatic glucosylation of human skin collagen. J. Clin. Invest. 67:1630-1635.

8. Kinoshita, J. H., S. Fukushi, P. Kador, and L. O. Merola. 1979. Aldose reductase in diabetic complications of the eye. Metab. Clin. Exp. 28(Suppl. 1):462-469.

9. Gabbay, K. H., L. O. Merola, and R. A. Field. 1966. Sorbitol pathway: presence in nerve and cord with substrate accumulation in diabetes. Science (Wash. DC). 151:209-210.

10. Gabbay, K. H., and J. H. Kinoshita. 1972. Mechanisms of development and prevention of cataracts. Isr. J. Med. Sci. 8:15571561.

11. Heath, H., and Y. C. Hamlett. 1976. The sorbitol pathway: effect of streptozotocin induced diabetes and the feeding of a sucroserich diet on glucose, sorbitol and fructose in the retina, blood and liver of rats. Diabetologia. 12:43-46.
12. Poulsom, R., R. P. Boot-Handford, and H. Heath. 1983. The effects of long-term treatment of streptozotocin-diabetic rats with an aldose reductase inhibitor. Exp. Eye Res. 37:507-515.

13. Tomlinson, D. R., J. Townsend, and P. Fretten. 1985. Prevention of defective axonal transport in streptozotocin-diabetic rats by treatment with "statil" (ICI 1228436), and aldose reductase inhibitor. Diabetes. 34:970-972.

14. Suárez, G., J. Novick, and A. L. Oronsky. 1984. Fed. Proc. 43:2022. (Abstr.)

15. Poulsom, P., D. J. Mirrlees, D. C. N. Earl, and H. Heath. 1983. The effects of an aldose reductase inhibitor upon the sorbitol pathway, fructose-1-phosphate and lactate in the retina and nerve of streptozotocin-diabetic rats. Exp. Eye Res. 36:751-760.

16. Stribling, D., D. J. Mirrlees, H. E. Harrison, and D. C. N. Earl. 1985. Properties of ICI 128, 436, a novel aldose reductase inhibitor, and its effects on diabetic complications in the rat. Metab. Clin. Exp. 34:336-344.

17. Woessner, J. F., Jr. 1961. The determination of hydroxyproline in tissue and protein samples containing small proportions of this imino acid. Arch. Biochem. Biophys. 93:440-447.

18. Chandrakasan, G., D. A. Torchia, and K. A. Piez. 1976. Preparation of intact monomeric collagen from rat tail tendon and skin and the structure of the nonhelical ends in solution. J. Biol. Chem. 251:6062-6067.

19. Chung, E., and E. J. Miller. 1974. Collagen polymorphism: characterization of molecules with the chain composition [1(III) $]_{3}$ in human tissues. Science (Wash. DC). 183:1200-1201.

20. Li, W., M. Khatami, and J. H. Rockey. 1985. The effects of glucose and an aldose reductase inhibitor on the sorbitol content and collagen synthesis of bovine retinal capillary pericytes in culture. Exp. Eye Res. 40:439-444.

21. Bitensky, M. W., A. Kowluru, and R. A. Kowluru. 1986. Increased cross linking of proteins in diabetes. Fed. Proc. 45:1837. (Abstr.)

22. Ahmed, M. U., S. R. Thorpe, and J. W. Baynes. 1986. Identification of $N$-carboxymethyllysine as a degradation product of fructoselysine in glycated protein. J. Biol. Chem. 261: 4889-4894.

23. Eaton, R. P., W. L. Sibbitt, and A. Harsh. 1985. The effect of an aldose reductase inhibiting agent on limited joint mobility in diabetic patients. J. Am. Med. Assoc. 253:1437-1440.

24. Rosenbloom, A. L., J. H. Silverstein, D. C. Lezotte, K. Richardson, and M. McCallum. 1981. Limited joint mobility in childhood diabetes mellitus indicates increased risk for microvascular disease. $N$. Engl. J. Med. 305:191-194.

25. Eaton, R. P. 1986. Aldose reductase inhibition and the diabetic syndrome of limited joint mobility: implications for altered collagen hydration. Metab. Clin. Exp. 35(Suppl. 1):119-121.

26. Eaton, R. P. 1986. The collagen hydration hypothesis: a new paradigm for the secondary complications of diabetes mellitus. $J$. Chronic Dis. 39:763-766.

27. Tomlinson, D. R., G. B. Willars, and J. P. Robinson. 1986. Prevention of defects of axonal transport in experimental diabetes by aldose reductase inhibitors. Drugs. 32(Suppl. 2):15-18.

28. Morrison, A. D., R. S. Clements, Jr., and A. I. Winegrad. 1972. Effects of elevated glucose concentrations on the metabolism of the aortic wall. J. Clin. Invest. 51:3114-3123.

29. Lorenzi, M., S. Toledo, G. R. Boss, M. J. Lane, and D. F. Montisano. 1987. The polyol pathway and glucose 6-phosphate in human endothelial cells cultured in high glucose concentrations. Diabetologia. 30:222-227.

30. Ham, A. W. 1974. Histology. 7th ed. J. B. Lippincott, Philadelphia. 598.

31. Monnier, V. M., V. Vishwanath, K. E. Frank, C. A. Elmets, P. Dauchot, and R. R. Kohn. 1986. Relation between complications of type I diabetes mellitus and collagen-linked fluorescence. $N$. Engl. J. Med. 314:403-408. 